\title{
Heavy Metals Nanofiltration Using Nanotube and Electric Field by Molecular Dynamics
}

\author{
Tiago da Silva Arouche, ${ }^{1}$ Rosely Maria dos Santos Cavaleiro, ${ }^{1,2}$ \\ Phelipe Seiichi Martins Tanoue, ${ }^{1}$ Tais Sousa de Sa Pereira, ${ }^{1}$ Tarciso Andrade Filho, ${ }^{3}$ \\ and Antonio Maia de Jesus Chaves Neto $\mathbb{D}^{1,4,5}$ \\ ${ }^{1}$ Laboratory of Preparation and Computation of Nanomaterials (LPCN), Federal University of Pará, C. P. 479, 66075-110 Belém, \\ PA, Brazil \\ ${ }^{2}$ Pos-Graduate Program in Engineering of Natural Resources of the Amazon, ITEC, Federal University of Pará, C. P. 2626, 66.050- \\ 540 Belém, PA, Brazil \\ ${ }^{3}$ Universidade Federal do Sul e Sudeste do Pará, Campus de Marabá. FL 17, QD 04, LT Especial, 68505080 Marabá, PA, Brazil \\ ${ }^{4}$ Pos-Graduation Program in Chemical Engineering, ITEC, Federal University of Pará, C. P. 479, 66075-900 Belém, PA, Brazil \\ ${ }^{5}$ Science Faculty, ICEN, Federal University of Pará, C. P. 479, 66075-900 Belém, PA, Brazil
}

Correspondence should be addressed to Antonio Maia de Jesus Chaves Neto; amchaves@ufpa.br

Received 7 February 2020; Revised 9 April 2020; Accepted 15 April 2020; Published 11 May 2020

Academic Editor: Renyun Zhang

Copyright (C) 2020 Tiago da Silva Arouche et al. This is an open access article distributed under the Creative Commons Attribution License, which permits unrestricted use, distribution, and reproduction in any medium, provided the original work is properly cited.

\begin{abstract}
Heavy metal contamination in the world is increasing the impact on the environment and human life. Currently, carbon nanotubes and boron are some possible ideals for the nanofiltration of heavy metals due to the property of ion selectivity, optimized by the applications of the surface and the application of an external electric field. In this work, molecular dynamic was used to transport water with heavy metals under the force exerted by the electric field action inside nanotubes. This external electric field generates a propelling electrical force to expel only water molecules and retain ions. These metal ions were retained to pass through only water molecules, under constant temperature and pressure, for a time of $100 \mathrm{ps}$ under the action of electric fields with values from $10^{-8}$ to $10^{-1}$ au. Each of the metallic contaminants evaluated $\left(\mathrm{Pb}^{2+}, \mathrm{Cd}^{2+}, \mathrm{Fe}^{2+}, \mathrm{Zn}^{2+}, \mathrm{Hg}^{2+}\right)$ was subjected to molecular test simulations in the water. It was found that the measurement of the intensity of the electric field increased or the percentage of filtered water reduced (in both nanotubes), in which the intramolecular and intermolecular forces intensified by the action of the electric field contribute to retain the heavy metal ions due to the evanescent effect. The best results for nanofiltration in carbon and boron nanotubes occur under the field $10^{-8}$ au. Since the filtration in the boron nitride nanotubes, a small difference in the percentage of filtered water for the boron nitride nanotube was the most effective (90 to $98 \%$ ) in relation to the carbon nanotube (80 to $90 \%$ ). The greater hydrophobicity and thermal stability of boron nanotubes are some of the factors that contributed to this result.
\end{abstract}

\section{Introduction}

The term heavy metals (HM) is used to classify a group of highly reactive molecules, toxic to the environment and human life [1]. Even though it is a consolidated term, it has been questioned for bringing together elements with different chemical properties and reactivity [2]. Among the most harmful and frequent elements found in the environment above the permitted limits are copper, mercury, chromium, lead, manganese, cadmium, nickel, zinc, and iron [3].
Many of the HMs are naturally present in the environment, associated with other minerals and in rocks, but the distribution of HM can be altered by forces of nature such as floods and soil erosion; however, anthropic activities are mainly responsible by increasing the levels of these pollutants in the soil [4,5], water [6], and atmosphere [7]. The urbanization and industrialization are the main causes of the increase of the concentration of HM [8] and reached a stage in which society and the scientific community are no longer able to control and predict its real impact $[9,10]$. The 
effluents generated by industries, mining, agriculture, hospitals, and laboratories must receive treatment on the places where they are generated before disposal in sewage networks to eliminate the risk of contamination of water and soil [11, 12]. Contaminated effluent water can be absorbed by plants and animals, causing poisoning at all levels of the food chain [13]. The soil and sediments favor the adsorption and permanence of metals, while water is a transition environment and facilitates part of the chemical reactions, transport, and diffusion of metals [14-16].

Nanotube-based water purification devices have the potential to transform the desalination and demineralization field through their ability to remove salts and HM [17] without significantly affecting the fast flow of water molecules [18]. In the environment, the high reactivity of HM ions facilitates the formation of new compounds with organic molecules or metals and thus promote their persistence in soils and water [19]. There are new compounds with HM basis that have been used as food for many species, and because they are foreign to metabolism, they are not expelled out. When man feeds on contaminated vegetables and animals or consumes contaminated water by them, the HM accumulates in the body and will cause serious diseases through progressive bioaccumulation [20,21].

Molecular dynamics (MD) allows the nanoscale computational study of macrosystems, analyzing the interactions between atoms as a function of time [22, 23], and has contributed to the development of new technologies for protection and conservation of the environment. Thus, through the integration of the equations of motion described by Newtonian mechanics [24], the movements of atoms, which occur at different time scales, are continuously registered considering the intermolecular and intramolecular forces that act on them in their trajectories [25].

The selective transport of HM ions in aqueous media has been analyzed in studies of MD simulations [26, 27] and has potential use in effluent nanofiltration (NF). Simulation studies of the MD of transporting solid molecules in water using carbon nanotubes (CNT) and boron nitride nanotubes (BNNT) have contributed in several areas of knowledge, such as medicine and current processes desalination $[28,29]$. In recent years, research with BNNT has revealed the superiority of some properties over CNT, such as the extremely high resistance to torsion [30], the selectivity for ion separation [31,32], and the conductivity and thermal stability at high temperatures. Such advantages lead to the hypothesis that BNNT is an adequate substitute for CNTs, but studies on the biocompatibility for the use of BNNT have not been conclusive [33].

Previous simulations revealed that the adsorption capacity of electrically charged solid particles is improved by using the nanotube surface modification or by applying an external electric field (EF) [34-36]. EF increases the reactivity of HM inside the nanotube and increases the adsorption capacity of these ions to CNT and BNNT $[37,38]$. The application of electric cap in BNNT for NF of pollutants [39] and desalination [40] is also a reality. Studies with BNNT are more recent than studies with CNT and even with better performance on CNT; more research is needed to better evaluate the possible biocompatibility of this nanostructure [41, 42].

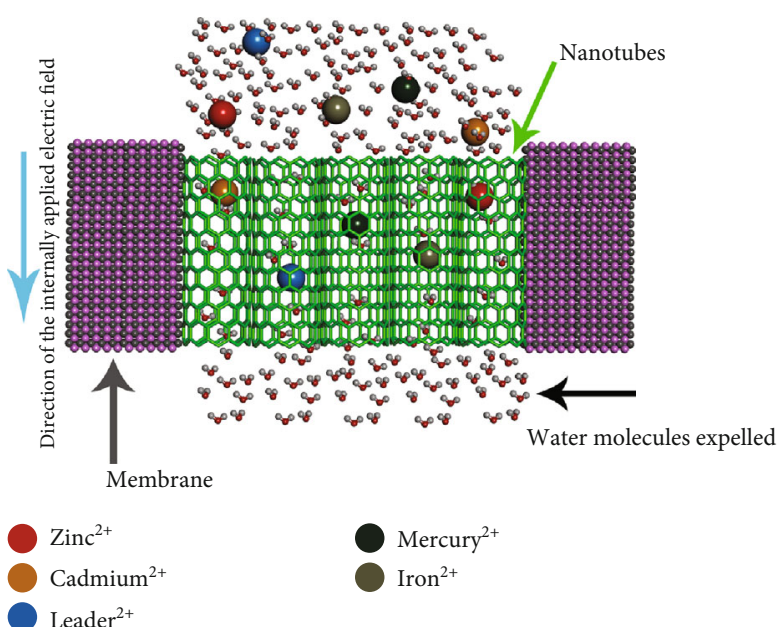

(a)

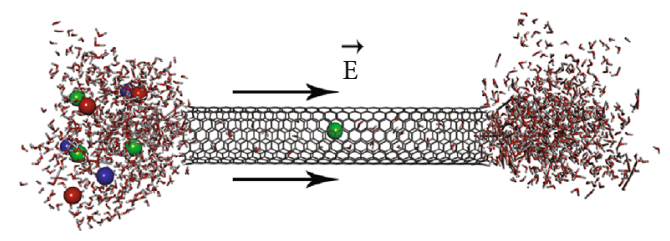

(b)

Figure 1: Nanofiltering: (a) near real model and (b) simplified model for simulation under EF.

In this work, we present a computational modeling study analyzing the MD of HM filtration in water, in two simulation phases: in CNTs and BNNT, both under the effect of constant EF, longitudinal, and external to the single-walled type nanotubes, where we have the system close to the real model in Figure 1(a). Also, we have our computational model in Figure 1(b). In this study, it was observed that the effect caused by the rotation of the metal molecules around the NT produces an evanescent field [43]. This work aimed to use CNT and single-walled BNNT to make an NC that removes HM ions from the water, through the action of a uniform EF. The MD simulation allowed the understanding of the interaction properties of $\mathrm{HM}$ for the: $\mathrm{Pb}^{2+}, \mathrm{Hg}^{2+}$, $\mathrm{Fe}^{2+}, \mathrm{Zn}^{2+}$, and $\mathrm{Cd}^{2+}$ ions in water, and it was observed that both nanotubes are effective in the NC of these HM in water under the electric field $10^{-8}$ au.

\section{Literature Review}

The contamination of living beings by HM is increasing and harmful to the metabolism of all species in the world [44]. The poorest countries have more pollution due to higher HM due to the lack of basic sanitation and the disposal of untreated effluents in the environment. [45].

Among the 35 known types of metals, 23 elements have an atomic radius between $63.546 \AA$ and $200.590 \AA$, with a density greater than $4.0 \mathrm{~g} / \mathrm{cm}^{3}$, chemical criteria to be classified as HM. In this category, in addition to chemical properties, these metals have a gloss, excellent heat conductivity, and high melting and boiling points [46] This group of inorganic elements, in any concentration, is highly toxic 
and other HMs need control to not exceed the minimum concentrations allowed in the environment and living organisms [44].

Part of HM are toxic to the human body metabolism such as lead, considered a major environmental threat, since it is found in the air, water, dust, and soil [47] and in several products used by man, without the population knowing the risks of its manipulation and the correct disposal [48]. There is still no safe level of exposure to lead, an extremely toxic heavy metal, and the biggest victims of poisoning are developing children and pregnant women [49]. Children poisoning is irreversible, chronic and cumulative, impairs cognitive impairment, and school and work performance. [50].

Some HM participates in human metabolism in minimal concentrations and is called trace elements. In this class are copper, cobalt, iron, nickel, magnesium, molybdenum, chromium, selenium, manganese, and zinc. These metals must not exceed the plasma concentration limit to avoid the risk of acute or chronic intoxication [51].

The daily requirement for copper ranges from 50 to $120 \mathrm{mg}$, with $80 \mathrm{mg}$ being the ideal average for a $70 \mathrm{~kg}$ adult. Some tissues require more copper, such as the liver, brain, spleen, bone and skeletal muscle [52], the liver, and spleen being considered reserve organs. Minimal concentrations of copper are essential for the metabolic functions of copperdependent enzymes cuproenzymes, such as cytochrome $\mathrm{C}$ oxidase, cytosolic superoxide dismutase, lysyl oxidase, tyrosinase, ceruloplasmin, and dopamine $\beta$-hydroxylase [20]. These enzymes catalyze physiological reactions related to oxidative phosphorylation, inactivation of free radicals, collagen and elastin biosynthesis, melanin formation, blood clotting, iron metabolism, and catecholamine synthesis [53], zinc activates immune responses in children and adolescents, and vanadium contributes to regulating insulin activity in glucose metabolism; however, these metals must be within the established limits to be beneficial to health [54].

Excessive HM in the body causes endocrine interference, mental disorders, damage blood cells, and respiratory, hepatic, and renal diseases that compromise the vitality of all systems $[55,56]$. The progressive accumulation of HM results in neuromuscular slowness that progresses as a degenerative process that simulates states of dementia or the symptoms of Parkinson's and Alzheimer's disease [57, 58]. In the long run, the accumulation of MP or its compounds destroys nucleic acids, causes mutations, behavioral disorders (autism, attention deficit and hyperactivity, aggressiveness), heart disease, kidney disease, and cancer and interferes with the reproductive process [51].

Keeping the environment balanced concerning the toxicity of HM, it is necessary to seek the chemical safety of the environment, achieved by carrying out processes to ensure the safety of human health and the environment [52]. The WHO International Chemical Safety Program (ICSP) [44] establishes scientific bases for the management of chemical products and strengthens safety standards for natural elements, as well as covering all situations of exposure, considering the natural occurrence in the environment until extraction or synthesis, industrial production, use, reuse, and disposal [59].
The use of single-walled CNT or multiple-walled to filter $\mathrm{HM}$ ions in aqueous solutions is efficient in the NF processes of other pollutants [60-63]. Among the sensors used to detect HM [35, 36], some have high sensitivity to identify the kind and level of contamination, based on a threedimensional hybrid electrode system in CNT [64]. After $\mathrm{HM}$ or its compounds are detected, separation methods will be chosen, such as the use of chemical precipitation [65], reverse osmosis [31], filtration [45], adsorption on activated carbon [66], the use of adsorbents such as aluminosilicates $[67,68]$, or processes of oxy-reduction [69].

CNT and single-walled BNNT are nanostructures used for the NC of HM ions in aqueous solutions in MD studies and have shown good results [30]; however, the performance of the BNNT has surpassed the CNTs in some properties. BNNTs have all the properties of NTC and also excellent chemical inertness and superior thermal stability [70]. Mixed with polymers, BNNTs can be used to reinforce synthetic fibers, exceeding the resistance of the same fiber by $35 \%$ when reinforced with CNT; however, many studies are still needed to better understand the stresses at the interface of polymers with these two kinds of nanotubes [71]. In medicine, the use of CNTs is well established as a biomaterial, whereas, for BNNT, biocompatibility has not yet been confirmed [33].

2.1. Nanofiltration. NF membranes used to recycle wastewater, require high flow and energy rates during the NF process [66]. Reverse osmosis is the ideal separation method for ion removal [67], but it does not remove monovalent ions. NF can distinguish molecules based on their size or valence [72] and is often used to remove bi- or trivalent ions from water because they represent part of "water solids." The main disadvantage of membrane NF lies in the blocking of pores (initiated with organic molecules that incorporate other molecules and prevent NF), a process called fouling $[73,74]$. This block reduces the flow of matter and reduces the life of the membrane, requiring mechanical or chemical cleaning [75, 76]. The separation process combined with the addition of an adjustable EF to the NF membrane was first investigated and denoted as electron filtration (EFT) [77] and brings together two driving forces: pressure and EF used mainly for the separation of charged molecules or particles electrically [78]. EFT works by trapping contaminants loaded in the membrane pores with the help of EF. The flow of electroosmosis generated by the superimposed EF also contributes to the improved permeate flow [60]. The ionic currents through the membranes depend on the movement of the ions through the pores of the membrane, a process called permeation. The way water is transported in nanotubes can be predicted with the use of EF, which influences the electrical dipoles in water molecules giving them a direction [79].

In order to understand the process of simulating the $\mathrm{NC}$ of water inside the nanotubes, it is necessary to understand that the nanotube must have enough diameter to allow a greater flow of water molecules, which also facilitates the transport of the solid molecules of the simulation, without friction on the walls of the CNT [80]. The direction taken by the water molecules inside the CNTs is a phenomenon induced by the application of external and longitudinal EF 
to the NT that increases the speed of the flow internally (since it acts on a system that also has a charge) and drives the molecules of water in the direction of the positive pole with an electrical force acting on the HM ions due to the EF where the electrostatic attraction would normally direct them, due to the electronegativity of oxygen [81].

The complexity of the interactions of hydrogen bonds in water [82] and the impossibility of carrying out real experiments with water in high-intensity electric fields $[83,84]$ are some of the reasons that require the advancement of simulation studies in the field of MD.

In MD simulations, the stoichiometry of hydrogen bonds and molecular orientations undergo changes due to increased temperatures or increased intensities of the electric fields [85], and these hydrogen bonds can stabilize molecules whose electrical dipoles are oriented perpendicularly to the direction of the EF [86]. Molecular systems present movements that occur at different time scales, considering both intermolecular and intramolecular forces. The effect caused by the rotation of the molecules around the nanotubes produces an evanescent field [87], such an effect occurs on the walls of the nanotube. EF does not propagate as an electromagnetic wave, but its activity is spatially concentrated on the walls of the nanotube, also functioning as a "force trap" or valve. The trajectories of ions in nanotubes can be quite complex, and the effect of applied EF can trap electrically charged ions [88].

2.2. Materials and Methods. In order to perform the computer simulations for this study, the both single-walled armchair structures of the CNT and BNNT were first modeled, because this structure has a better capacity to conduct energy. The CNT was modeled with 1064 atoms, 91.052 $\AA$ in length and $10.081 \AA$ in diameter; the BNNT received modeling with 1162 atoms, measured in length $91,985 \AA$ and the diameter measured 12.691 $\AA$. It is hard to build two nanotubes of different nature with the same diameter and length. We compared the results in a very general way of the NF of the two nanotubes due to the percentage difference of $20.6 \%$ in these diameters.

HM ions and water molecules were modeled in the Gaussian 09 software. Then, the conformational analysis was performed in the Hyperchem 7.5 software when the studied molecules go through different conformations to minimize the energy of each molecule. Interactive cycles in energy minimization were applied individually, for each molecular structure [89]. The convergence criterion of the energy gradient was $0.01 \mathrm{kcal} /(\mathrm{mol} \AA)$.

We use the methodology of molecular mechanics together with the processes and parameterizations of the force fields and molecule connections. The system consists of 100 water molecules for each ion present, 80 simulations were performed, 40 molecules of water for NTC, and 40 molecules of water for BNNT. The intermolecular forces have been described in terms of potential energy, kinetic, and thermal capacity functions, as well as lengths and angles of hydrogen bonds and nonbinding interactions. After the energy minimization, the conformational analysis [90] of the molecules was carried out, where a systematic search was made for the values of the dihedral angles of all the rotatable connections [91], to explore the conformational space of the molecules and so find a lower energy arrangement.

Once more stable molecular structures were obtained, MD calculations were made, based on the MM+force field, calculations of the interaction energies, and Newton's equations for motion. The equations were used to predict the position and velocity of all atoms at each time interval. Thus, $\mathrm{MD}$ was carried out with the system at an approximate temperature of $300 \mathrm{~K}$, varying slightly during the simulation but controlled by the NPV ensemble $[91,92]$. EF was applied longitudinally to the outer surface of the two kinds of nanotubes and with constant intensities in a vacuum, so that the nanotubes remain rigid during all the simulations, while the water molecules and ions relaxed.

The duration of each simulation was $100 \mathrm{ps,} \mathrm{and} \mathrm{they}$ were carried out under the action of eight uniform electric fields between $10^{-1}$ au and $10^{-8}$ au $(1 \mathrm{au}=5.14 \times 109 \mathrm{~V} / \mathrm{cm})$. The study allows the calculation of some physical properties such as kinetic energy (EKIN), potential energy (EPOT), and total energy (ETOT). The HM ions were $\mathrm{Hg}^{2+}, \mathrm{Fe}^{2+}, \mathrm{Zn}^{2+}$, $\mathrm{Cd}^{2+}$, and $\mathrm{Pb}^{2+}$. The ions of each heavy metal were placed, individually with the water molecules, inside the nanotubes. Each set of molecules was subjected to sixteen simulation processes, eight in the NTC and eight in the BNNT.

The simulation was performed using the similar $\mathrm{MD}$ methodology proposed by Yang et al. [77] and Neto et al. [79]. The MD method calculated the trajectory of the molecules when exposed to the electric fields of EF.

\section{Results and Discussion}

The MD simulation of HM NF in nanotubes of different molecular conformations made it possible to evaluate the influence of external EF on CNT and BNNT as well as possible interactions between HM ions, the inner surface of nanotubes and water molecules. Figure 1 demonstrates the NF process under the action of an EF that results in the retention of $\mathrm{HM}$ ions inside the nanotube, and the passage and reflux of water molecules, in the experiment, with high kinetic energy. The flow of water molecules is different from the molecules that pass through the interior and those that return towards the EF due to internal collisions with other water molecules, HM ions, and the nanotube wall. The favorable direction of the flow of water molecules under the EF is towards the positive pole of the nanotube due to the electronegativity of the oxygen atoms; however, the EF also increases the intermolecular interactions of the water, and thus the NF tends to be attracted to positive poles also contributes to this effect.

EF has an electrical force effect on the transport of water molecules in CNT and BNNT, but the influence of the size, chemical structure, and electrostatic potential of the molecules that makes up the system generates internal charges when the EF is applied. Nanotube simulations for studying the transport of solid molecules in water report the importance of the internal electrical charge in the total energy of the simulated system. The effect of trapping heavy metal ions is due to the intensity of the EF applied in interaction with 


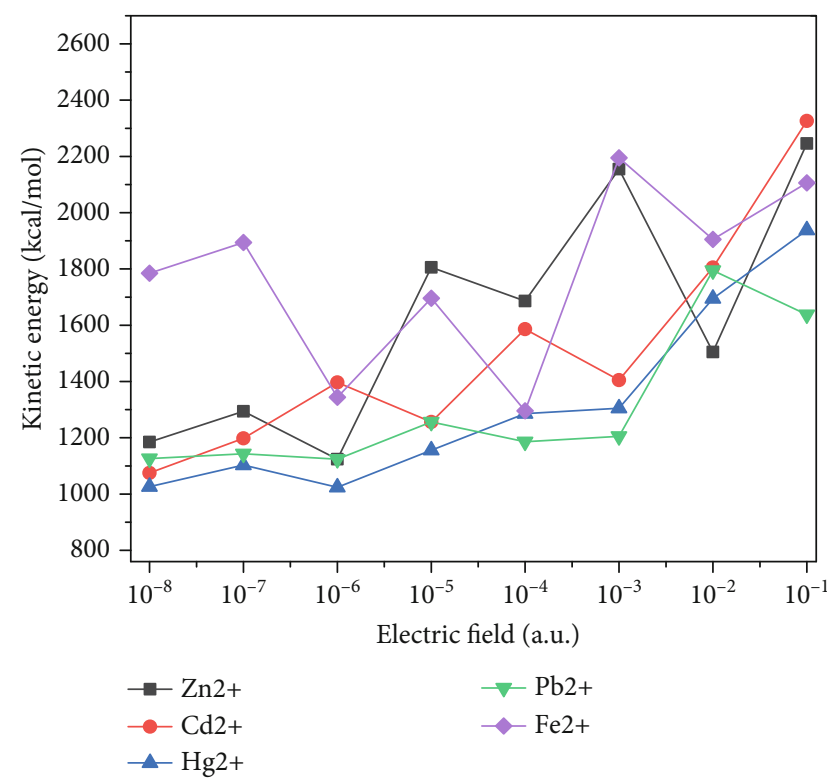

(a)

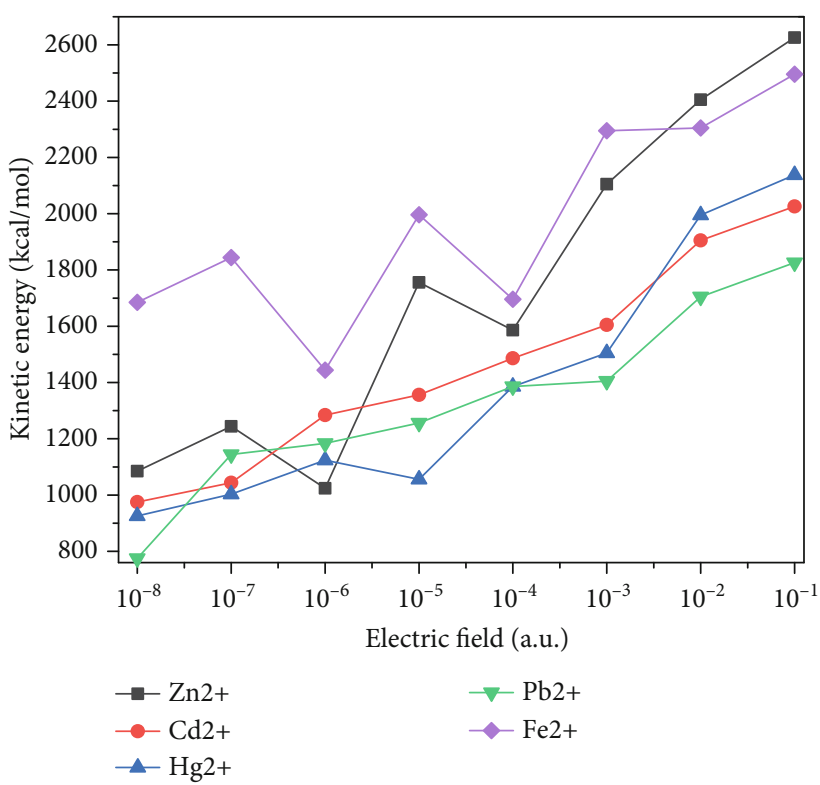

(b)

Figure 2: Kinetic energy of HM, under the action of EF in (a) CNT and (b) BNNT.

the intermolecular and intramolecular forces of the water molecules and HM ions. These forces are more intense in metal ions, good conductors of energy; in the water molecules closest to the nanotube walls, there is an evanescent effect, which temporarily retains these molecules next to the nanotubes [34, 35].

The effect of EF on NF in CNT and BNNT concerning the kinetic energy of HM ions is recorded in Figure 2(a) for CNT and Figure 2(b) for BNNT. The action of the electric field in the nanotube and molecules in the system during the simulation time was able to trap ions and few water molecules, causing most of them to be expelled from inside the nanotube with high values of velocities and kinetic energy. In general, in both nanotubes, the increase in kinetic energy was proportional to the intensity of the EF, but in both nanotubes for molecules with lower weight and atomic density (iron and zinc), they showed greater variations in kinetic energy and in potential energy. However, there are small variations in the linear growth of the kinetic energy, as in the case of the zinc $2+$ ion, due to the constant molecular rearrangement inside the nanotubes. Two molecules with higher weight and molecular density showed little variations in kinetic energy (lead and mercury). The kinetic energy of the metal ion (which has an electric charge) increased directly proportional to the EF, because it follows the classic electric force (electric force $=$ electric charge $\times \mathrm{EF}$ ).

The in situ temperature of the system was evaluated because the electric fields influence the thermal capacity of the molecules even when the NPT ensemble is applied, as they are variations of little intensity and are generally controlled by the system. These changes occur in the initial picoseconds of the simulation time. The in situ temperature of the HM ion simulation is displaying in Figure 3(a) for CNT and Figure 3(b) for BNNT. The heating capacity is divided by the amount of substance, mass, or volume; therefore, this value does not depend on the size or extension of the molecules and does not remain constant. Other system variables such as temperature and pressure were kept constant in this experiment. The thermal capacity varies with the application of EF, even though this field is uniform, as it causes an increase in the kinetic energy of the molecules that increases the total energy of the system. Thus, the increase in EF is a decisive factor in the systems in situ temperature. In CNTs, cadmium, iron, and lead had the highest temperatures in situ, followed by lead; these ions are the most electronegative in BNNT, lead, cadmium, and zinc.

Evaluating the transport of water molecules in CNT and BNNT, under the influence of the electric field, a sequence of 16 simulations with the same boundary conditions, without containing the HM ions, was performed, but without the presence of the metal ions (Figure 4). It seeks to know the percentage of water molecules filtered in the CNT and BNNT, to understand whether the HM molecules under the action of EF and the inner walls of the nanotubes influence the transport of confined water. We tried to evaluate the percentage of water molecules filtered under the influence of $\mathrm{EF}$ and van der Waals forces.

The dipole moment of water molecules allows the intensity of the EF to influence its flow in a direction $[80,93]$. The alignment of the water molecules induced by EF will cause the preexisting hydrogen bonds to become unstable and break. The balance between hydrogen bonds and van der Waals forces will give rise to dispersed molecular clusters, because EF decreases the lengths of the molecular bonds between oxygen and hydrogen, causing changes in the stability of water molecules, vibrational frequencies, and dissociation energy that influenced the percentage of molecules in both nanotubes. There was a better yield of NF in BNNT because even though both are hydrophobic, the chemical constitution of BNNT increases their degree of hydrophobicity, which 


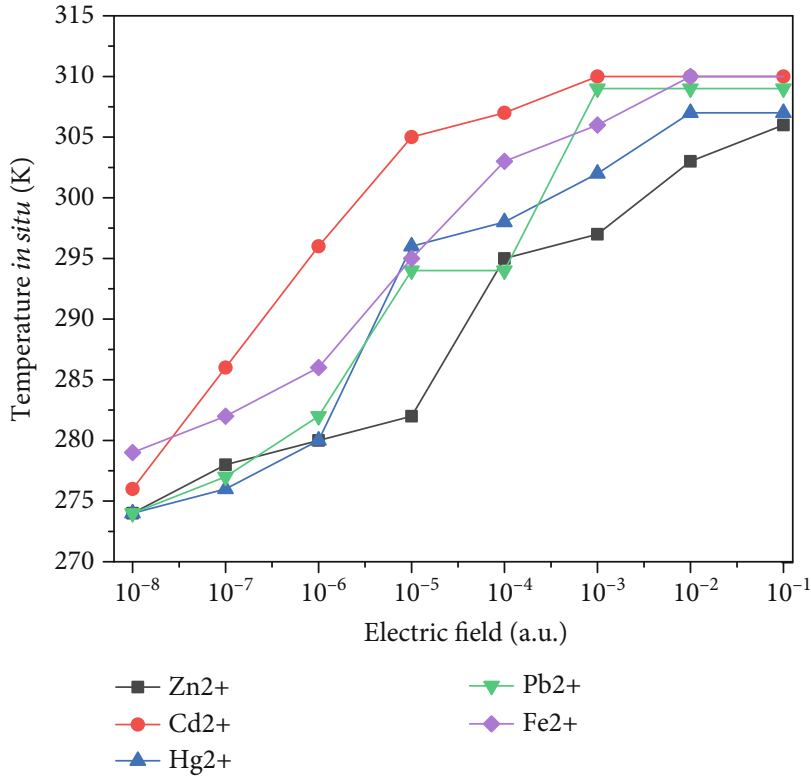

(a)

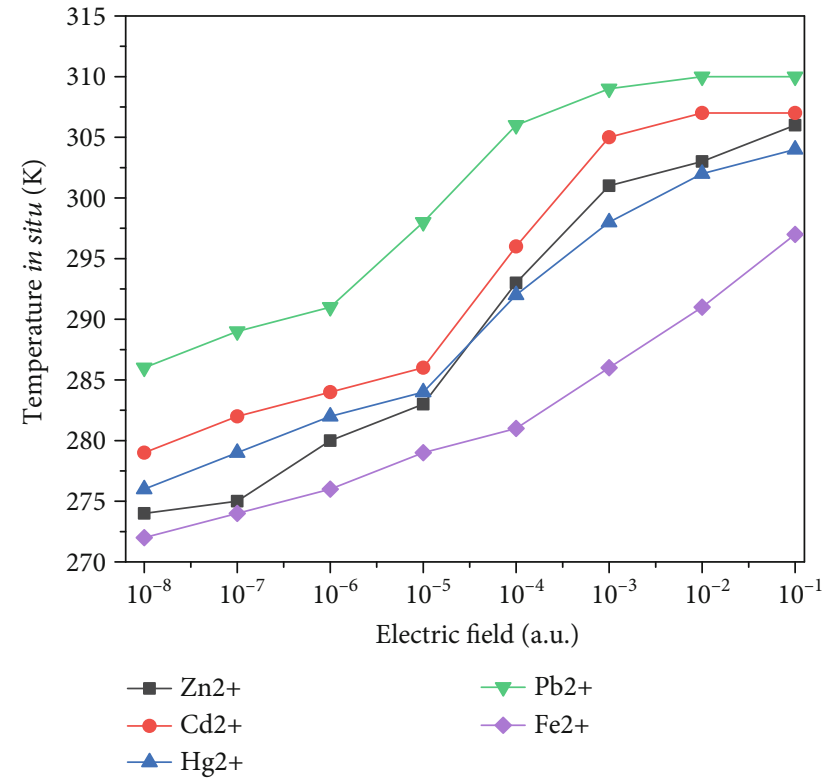

(b)

FIgure 3: In situ temperature versus EF in (a) NTC and (b) NBBT.

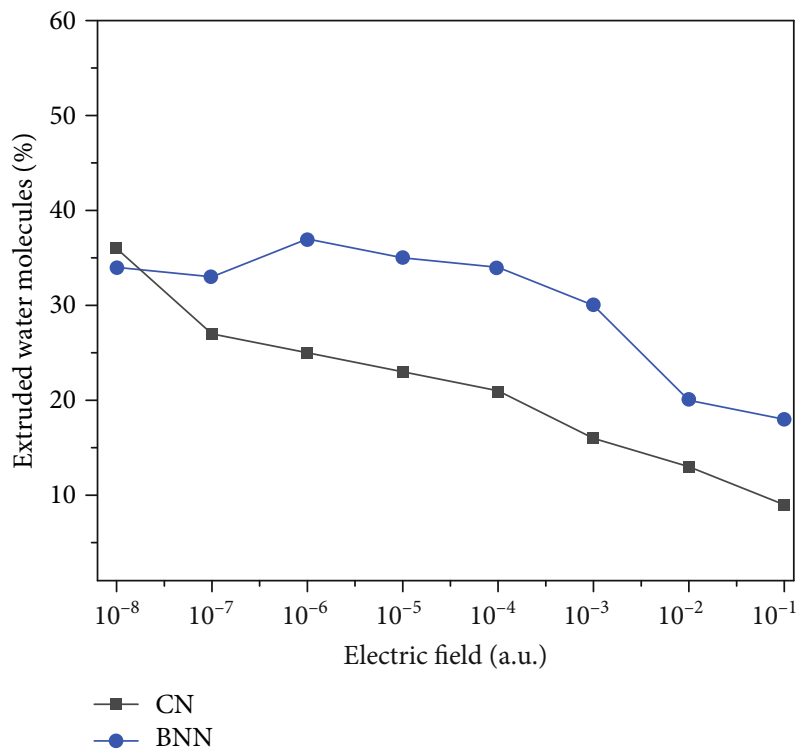

Figure 4: Percentage of water molecules expelled by CNT e BNNT without ions.

contributed to the expulsion of more water than in CNT [90]. The influence of EF on water dynamics, generating flows inversely proportional to the increase in EF, is well established in these conditions, as well as that the propelling effect of water in the transport of molecules inside the nanotube is related to size, chemical structure, and electrostatic potential of the molecules to be filtered.

The NF of the HM molecules in Figure 5(a) for CNT and Figure 5(b) for BNNT to confirm the influence of EF and the kind of nanotube structures (BNNT and CNT) used to remove water from HM ions; however, the results showed that both BNNT and CNTs are effective in retaining HM ions. The difference between them is made by the greater hydrophobicity and thermal conductivity of the BNNT. The EF effect on HM at $10^{-8}$ au is enough for filtration. In addition to implying lower energy consumption in systems that aim to separate persisting HM in wastewater after conventional NF, both nanotubes perform this separation; however, in the evaluated parameters, there was a better efficiency of NF of BNNT about CNTs, but the difference is not a cause of replacement of CNT by BNNT because both are effective. The nanotube option should also take into account the costs of an NF system budget.

The trapping of HM ions in modified NC membranes composed of CNTs was the subject of previous studies and concluded that the same metal ions examined were almost $100 \%$ trapped in NBBT [62]; in this study, there was a better performance of the $\mathrm{Cd}^{2+}$ ion [5]. The CNTs, due to its properties that keep it neutral and temporarily charged with the use of NF, showed better trapping in the $\mathrm{Pb}^{2+}$ ion, in the highest concentration examined. The metals caused a minimal decline in temperature flow and thermal capacity due to the incrustation of the membrane in the order of gravity.

The number of filtered water molecules was lower for CNT than BNNT due to the intermolecular forces present [94]. Both nanotubes have hydrophobic potential; however, BNNT has adsorption properties superior to CNT due to its greater transfer of electrons on the wall. The adsorption energy of HM in BNNT is directly related with the hydrophobicity of its molecules [95].

The effect of BNNT nanofiltration on $\mathrm{Pb}^{2+}$ ion is not good, because $\mathrm{Pb}$ is the heaviest and has little effect of the applied electrical force (electrical force $=$ electric charge $\times$ $\mathrm{EF})$. The removal effect is better when the electric field (EF) is $10^{-8} \mathrm{au}$, because the evanescence effect is smaller. Also, the greater the EF, the greater the evanescent effect is. The 


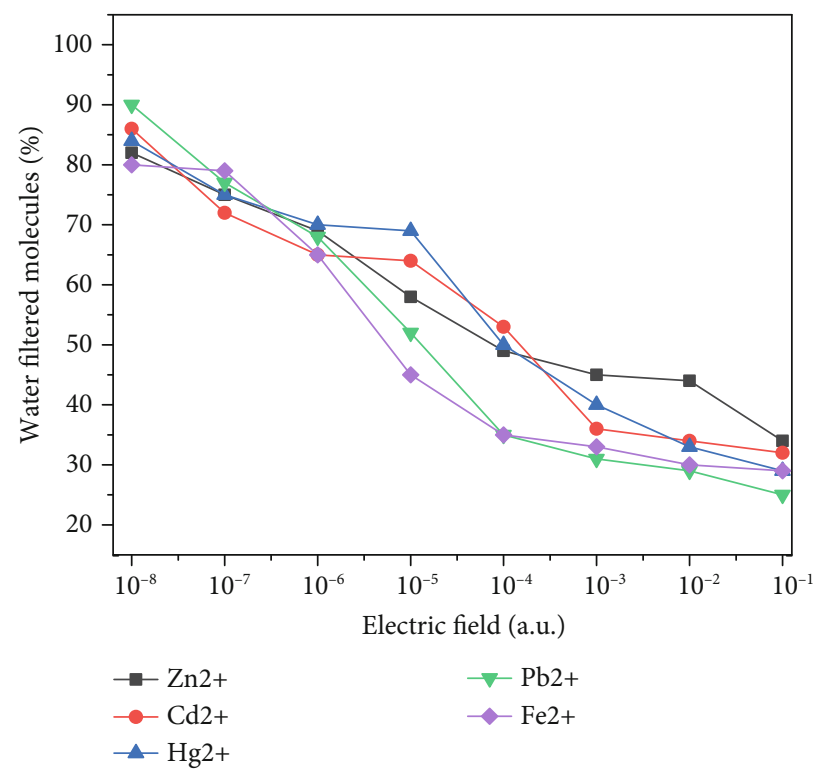

(a)

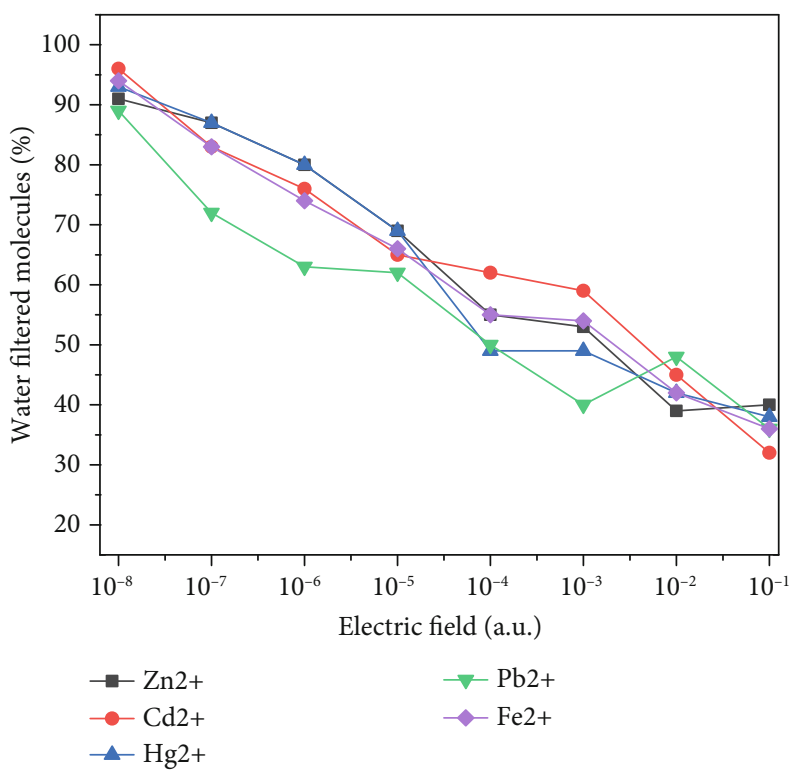

(b)

Figure 5: Percentage of water molecules expelled by NF: (a) inside CNT and (b) under EF inside BNNT.

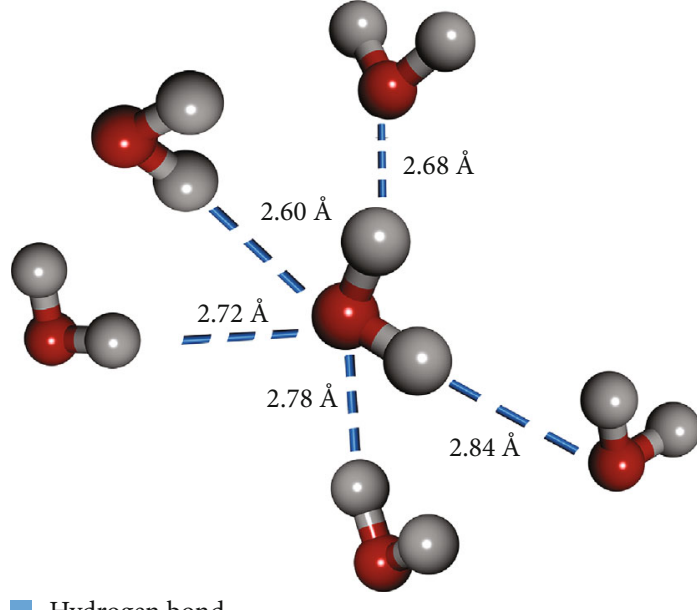

Hydrogen bond

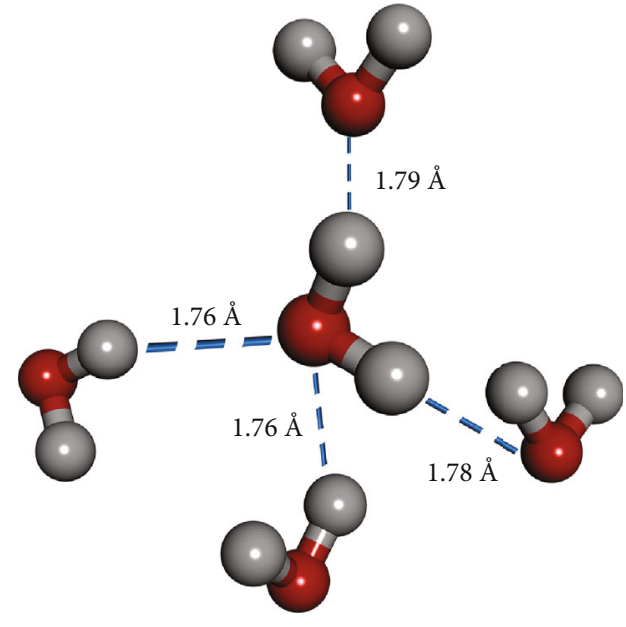

Hydrogen bond

(a)

(b)

FIGURE 6: Interatomic distance of hydrogen bonds in water confined: (a) without EF and (b) under EF.

evanescence effect produces the axial spin movement of all water molecules and HM inside the nanotube, thus decreasing the nanofiltration of water molecules and increase the kinetic energy to perform the axial turn inside the nanotube. The effect of EF on HM NF at $10^{-8}$ au EF is the best for HM filtration in this EF range.

Recent studies on confined water bring illuminating results on the influence of hydrogen bonds and the changes in this behavior in the presence of external electrical charges [96]. It is interesting to mention that hydrogen bonds account for a large part of the interactions of the water molecules. The hydrogen bonds in confined water, under EF, are different from the bonds established in free water because in the latter the bonds are stable and occur in a shorter distance between the atoms $[89,91]$ displayed in Figure 6.

The confined water $\mathrm{MD}$ is characterized by flexible hydrogen bonds [97] are unstable bonds (they quickly rupture and redox). The effects of the NT surface and water confinement requires that the $\mathrm{MD}$ simulations be carried out on NT with a diameter between $10 \AA$ to $12.2 \AA$ to facilitate the diffusion of water and friction on the internal surface of the NT [98]. Confined water needs minimal space to alternate the two geometric shapes of its molecular arrangements in closed and open chains [99].

Pure water is a poor conductor of electricity, but its free ions are attracted or attract other molecules. The EF, depending 
on the intensity, can cause electrolysis [100, 101]. Previous studies have shown that applying metallic electrodes to water, even at low intensity, causes interference in the orientation of water molecules and in the positioning of their atoms, which can be attracted or repelled. Similar orientations can occur on the surface of minerals containing alternating positive and negative charges, under the effect of electric fields.

Due to the partial covalence of hydrogen bonds in water, $\mathrm{HM}$ ions are easily distributed among their agglomerates under the action of EF increasing the intermolecular forces $[102,103]$. Both the carbon nanotube and the boron nitride have achieved higher levels of water flow compared to carbon nanotubes and are therefore expected to provide a more efficient water purification device.

\section{Conclusion}

The behavior of ionic compounds solubilized in water was analyzed, interacting with CNTs and BNNT under the action of EF. The trapping of HM ions in the NF process was effective in both nanotubes, but the intermolecular forces that act in the system allowed a different filtration flow, through filtered ions. The highest percentage of water molecules sequentially filtered in the NF of the ions was for CNT $\mathrm{Pb}^{2+}, \mathrm{Cd}^{2+}, \mathrm{Hg}^{2+}, \mathrm{Zn}^{2+}$, and $\mathrm{Fe}^{2+}$. In the case of BNNT, they were $\mathrm{Cd}^{2+}, \mathrm{Fe}^{2+}, \mathrm{Hg}^{2+}, \mathrm{Zn}^{2+}$, and $\mathrm{Pb}^{2+}$. In this simulation, BNNT demonstrated thermal and chemical stability in EF applications superior to that of CNT. Differences were found in the thermodynamic properties of CNT and BNNT, such as kinetic and potential energy, in addition to the increase in the thermal capacity of the system, where BNNT showed greater stability in all parameters than CNT. The evanescent effect was observed close to the inner walls of CNT and BNNT, resulting from the interactions of the intensity of the EF and the internal electrical charges of the molecules. The evanescence effect produces the axial spin movement of all water molecules and HM inside the nanotube, thus decreasing the nanofiltration of water molecules and increase the kinetic energy to perform the axial turn inside the nanotube. This effect temporarily retains water molecules close to the nanotubes. Ion capture by CNT is related to the effect of an evanescent attractive potential. Also, the greater the EF, the greater the evanescent effect is. Thus, probably, the nanofiltration would possibly get a better value to small EF. The results of this study can contribute as a theoretical basis for the development of an NF membrane system for the removal of $\mathrm{HM}$ ions from contaminated waters.

\section{Data Availability}

The data used to support this study are included within the article.

\section{Conflicts of Interest}

The authors declare that there is no conflict of interest regarding the publication of this paper.

\section{Authors' Contributions}

All authors designed and developed the study. All authors read and approved the final version of the manuscript.

\section{Acknowledgments}

The authors acknowledge the support of the Dean of Research and Graduate Studies (PROPESP) of the Federal University of Para (UFPA), Coordination for the Improvement of Higher Education Personnel (CAPES), National Council for Scientific and Technological Development (CNPq), and also to the Laboratory of Preparation and Computation of Nanomaterials (LPCN) of Prof. Dr. Antonio M. J. C. Neto for his contribution and inspiration through his work entitled Heavy metals nanofiltration using nanotube and electric field by molecular dynamics, exhibited at Electrochem 2019 conference, Glasgow.

\section{Supplementary Materials}

Table 1: Numerical values of the CNT kinetic energy (Kcal/mol) (Figure 2(a)). Table 2: Numerical values of the BNNT kinetic energy (Kcal/mol) (Figure 2(b)). Table 3: CNT in situ temperature (K) table referring to numerical values of the Figure 3(a). Table 4: BNNT in situ temperature $(\mathrm{K})$ numerical values of the Figure $3(\mathrm{~b})$. Table 5: Expulsion values of the NTC of the Figure 5(a). Table 6: Expulsion values of the BNNT to the Figure 5(b). Table 7: Percentage of water molecules expelled without adding ions (Figure 4). (Supplementary Materials)

\section{References}

[1] A. W. Tadesse, T. Gereslassie, Q. Xu, X. Tang, and J. Wang, "Concentrations, distribution, sources and ecological risk assessment of trace elements in soils from Wuhan, Central China," International Journal of Environmental Research and Public Health, vol. 15, no. 12, article 2873, 2018.

[2] A. Jose and J. G. Ray, “Toxic heavy metals in human blood in relation to certain food and environmental samples in Kerala, South India," Environmental Science and Pollution Research, vol. 25, no. 8, pp. 7946-7953, 2018.

[3] G. A. Engwa, P. U. Ferdinand, F. N. Nwalo, and M. N. Unachukwu, "Mechanism and health effects of heavy metal toxicity in humans," in Poisoning in the Modern World - New Tricks for an Old Dog?, 2019.

[4] L. Bücker-Neto, A. L. S. Paiva, R. D. Machado, R. A. Arenhart, and M. Margis-Pinheiro, "Interactions between plant hormones and heavy metals responses," Genetics and Molecular Biology, vol. 40, no. 1, Supplement 1, pp. 373-386, 2017.

[5] Z. L. He, X. E. Yang, and P. J. Stoffella, "Trace elements in agroecosystems and impacts on the environment," Journal of Trace Elements in Medicine and Biology, vol. 19, no. 2-3, pp. 125-140, 2005.

[6] M. Al-Abyadh, A.-Q. Mko, B. As, A.-A. Mm, and A.-K. Ag, "The effects of lead, cadmium, mercury and arsenic on fish and seawater in Red Sea and the Gulf of Aden at three different locations in Yemen OPEN ACCESS," SF Journal of Pharmaceutical and Analytical Chemistry, vol. 1, 2018. 
[7] M. A. Tofighy and T. Mohammadi, "Adsorption of divalent heavy metal ions from water using carbon nanotube sheets," Journal of Hazardous Materials, vol. 185, no. 1, pp. 140-147, 2011.

[8] K. Rehman, F. Fatima, I. Waheed, and M. S. H. Akash, "Prevalence of exposure of heavy metals and their impact on health consequences," Journal of Cellular Biochemistry, vol. 119, no. 1, pp. 157-184, 2018.

[9] H. Ali, E. Khan, and I. Ilahi, "Environmental chemistry and ecotoxicology of hazardous heavy metals: Environmental persistence, toxicity, and bioaccumulation," Journal of Chemistry, vol. 2019, Article ID 6730305, 14 pages, 2019.

[10] R. A. Bernhoft, "Cadmium toxicity and treatment," The Scientific World Journal, vol. 2013, Article ID 394652, 7 pages, 2013.

[11] N. Johri, G. Jacquillet, and R. Unwin, "Heavy metal poisoning: the effects of cadmium on the kidney," BioMetals, vol. 23, no. 5, pp. 783-792, 2010.

[12] X. Wang, Y. Sun, S. Li, and H. Wang, "Spatial distribution and ecological risk assessment of heavy metals in soil from the Raoyanghe Wetland, China," PLoS One, vol. 14, no. 8, article e0220409, 2019.

[13] L. Järup, "Hazards of heavy metal contamination," British Medical Bulletin, vol. 68, no. 1, pp. 167-182, 2003.

[14] M. Gavrilescu, K. Demnerová, J. Aamand, S. Agathos, and F. Fava, "Emerging pollutants in the environment: present and future challenges in biomonitoring, ecological risks and bioremediation," New Biotechnology, vol. 32, no. 1, pp. 147156, 2015.

[15] F. X. Kong, H.-w. Yang, X.-m. Wang, and Y. F. Xie, “Assessment of the hindered transport model in predicting the rejection of trace organic compounds by nanofiltration," Journal of Membrane Science, vol. 498, pp. 57-66, 2016.

[16] J. Wei, M. Duan, Y. Li, A. S. Nwankwegu, Y. Ji, and J. Zhang, "Concentration and pollution assessment of heavy metals within surface sediments of the Raohe Basin, China," Scientific Reports, vol. 9, no. 1, article 13100, 2019.

[17] L. Zhang, L. Jia, J. Zhang et al., "Understanding the effect of chemical modification on water desalination in boron nitride nanotubes via molecular dynamics simulation," Desalination, vol. 464, pp. 84-93, 2019.

[18] B. Corry, "Water and ion transport through functionalised carbon nanotubes: implications for desalination technology," Energy \& Environmental Science, vol. 4, no. 3, pp. 751-759, 2011.

[19] J. H. Duffus, “'Heavy metals' - a meaningless term? (IUPAC technical report)," Pure and Applied Chemistry, vol. 74, no. 5, pp. 793-807, 2002.

[20] H. K. Hussein, O. A. Abu-Zinadah, H. A. El-Rabey, and M. F. Meerasahib, "Estimation of some heavy metals in polluted well water and mercury accumulation in broiler organs," Brazilian Archives of Biology and Technology, vol. 56, no. 5, pp. 767-776, 2013.

[21] S. O. Ojoawo, A. Lateef, F. A. Oyeniran, O. T. Kupoluyi, O. S. Opatola, and J. O. Daramola, "Bioaccumulation of heavy metals in steel processing industrial effluents using Bacillus safensis LAU 13," Journal of Environment and Biotechnology Research, vol. 6, no. 1, pp. 58-63, 2017.

[22] D. J. Bonthuis, K. F. Rinne, K. Falk et al., "Theory and simulations of water flow through carbon nanotubes: prospects and pitfalls," Journal of Physics. Condensed Matter, vol. 23, no. 18 , article $184110,2011$.
[23] T. A. Beu, "Molecular dynamics simulations of ion transport through carbon nanotubes. II. Structural effects of the nanotube radius, solute concentration, and applied electric fields," The Journal of Chemical Physics, vol. 135, no. 4, article 044515, 2011.

[24] Y. Chan, S. L. Lee, W. Chen, L. Zheng, Y. Shi, and Y. Ren, "Newtonian flow inside carbon nanotube with permeable boundary taking into account van der Waals forces," Scientific Reports, vol. 9, no. 1, article 12121, 2019.

[25] G. Malloci, G. Serra, A. Bosin, and A. Vargiu, "Extracting conformational ensembles of small molecules from molecular dynamics simulations: ampicillin as a test case," Computation, vol. 4, no. 1, p. 5, 2016.

[26] R. Devanathan, D. Chase-Woods, Y. Shin, and D. W. Gotthold, "Molecular dynamics simulations reveal that water diffusion between graphene oxide layers is slow," Scientific Reports, vol. 6, no. 1, article 29484, 2016.

[27] M. Xie, L. D. Nghiem, W. E. Price, and M. Elimelech, "Relating rejection of trace organic contaminants to membrane properties in forward osmosis: measurements, modelling and implications," Water Research, vol. 49, pp. 265-274, 2014.

[28] S. Rikhtehgaran and A. Lohrasebi, "Water desalination by a designed nanofilter of graphene-charged carbon nanotube: a molecular dynamics study," Desalination, vol. 365, pp. 176$181,2015$.

[29] T. A. Hilder, D. Gordon, and S. H. Chung, "Salt rejection and water transport through boron nitride nanotubes," Small, vol. 5, no. 19, pp. 2183-2190, 2009.

[30] J. Garel, C. Zhao, R. Popovitz-Biro, D. Golberg, W. Wang, and E. Joselevich, "BCN nanotubes as highly sensitive torsional electromechanical transducers," Nano Letters, vol. 14, no. 11, pp. 6132-6137, 2014.

[31] W. F. Chan, H. Y. Chen, A. Surapathi et al., "Zwitterion functionalized carbon nanotube/polyamide nanocomposite membranes for water desalination," ACS Nano, vol. 7, no. 6, pp. 5308-5319, 2013.

[32] S. Joseph, R. J. Mashl, E. Jakobsson, and N. R. Aluru, "Electrolytic transport in modified carbon nanotubes," Nano Letters, vol. 3, no. 10, pp. 1399-1403, 2003.

[33] L. Horváth, A. Magrez, D. Golberg et al., "In vitro investigation of the cellular toxicity of boron nitride nanotubes," ACS Nano, vol. 5, no. 5, pp. 3800-3810, 2011.

[34] J. C. N. Aires, J. N. Cruz, R. P. Pantoja et al., "Carbon nanotube under an external uniform electric field using molecular dynamics acting as drugs sensor," Journal of Computational and Theoretical Nanoscience, vol. 15, no. 6, pp. 1971-1974, 2018.

[35] J. N. Cruz, E. S. Moraes, R. P. Pantoja, T. S. S. Pereira, G. V. S. Mota, and A. M. J. C. Neto, "Sensors using the molecular dynamics of explosives in carbon nanotubes under external uniform electric fields," Journal of Nanoscience and Nanotechnology, vol. 19, no. 9, pp. 5687-5691, 2019.

[36] E. C. Santos, A. F. G. Neto, C. E. Maneschy, J. Chen, T. C. Ramalho, and A. M. J. C. Neto, "A molecular dynamics of cold neutral atoms captured by carbon nanotube under electric field and thermal effect as a selective atoms sensor," Journal of Nanoscience and Nanotechnology, vol. 15, no. 5, pp. 3677-3680, 2015.

[37] H. J. Wang, A. L. Zhou, F. Peng, H. Yu, and L. F. Chen, "Adsorption characteristic of acidified carbon nanotubes for heavy metal $\mathrm{Pb}(\mathrm{II})$ in aqueous solution," Materials 
Science and Engineering A, vol. 466, no. 1-2, pp. 201-206, 2007.

[38] H. Wang, W. Wang, H. Wang, F. Zhang, Y. Li, and Z. Fu, "Urchin-like boron nitride hierarchical structure assembled by nanotubes- nanosheets for effective removal of heavy metal ions," Ceramics International, vol. 44, no. 11, pp. 12216-12224, 2018.

[39] M. Bodzek and M. Dudziak, "Elimination of steroidal sex hormones by conventional water treatment and membrane processes," Desalination, vol. 198, no. 1-3, pp. 24-32, 2006.

[40] J. Azamat, A. Khataee, and S. W. Joo, "Separation of a heavy metal from water through a membrane containing boron nitride nanotubes: molecular dynamics simulations," Journal of Molecular Modeling, vol. 20, no. 10, p. 2468, 2014.

[41] S. Mateti, C. S. Wong, Z. Liu et al., "Biocompatibility of boron nitride nanosheets," Nano Research, vol. 11, no. 1, pp. 334342, 2018.

[42] X. Li, L. Wang, Y. Fan, Q. Feng, and F. Z. Cui, "Biocompatibility and toxicity of nanoparticles and nanotubes," Journal of Nanomaterials, vol. 2012, Article ID 548389, 19 pages, 2012.

[43] J. C. N. Aires, A. F. G. Neto, C. E. Maneschy et al., "Molecular dynamics of $\mathrm{H}_{2}$ storage in carbon nanotubes under external electric field effects: a sensor proposal," Journal of Nanoscience and Nanotechnology, vol. 17, no. 7, pp. 4858-4863, 2017.

[44] European Commission DG ENV, Heavy Metals in Waste, Dep Environ Food Rural Aff, 2002.

[45] A. Azimi, A. Azari, M. Rezakazemi, and M. Ansarpour, "Removal of heavy metals from industrial wastewaters: a review," ChemBioEng Reviews, vol. 4, no. 1, pp. 37-59, 2017.

[46] S. Yanushkevich, S. C. Eastwood, M. Drahansky, and V. P. Shmerko, "We are Intech Open, the world' s leading publisher of Open Access books Built by scientists, for scientists TOP $1 \%$," Chapter: Mechanism and Health Effects of Heavy Metal Toxicity in Humans, vol. i, p. 13, 2016.

[47] A. T. Tiruneh, A. O. Fadiran, and J. S. Mtshali, "Evaluation of the risk of heavy metals in sewage sludge intended for agricultural application in Swaziland," International Journal of Environmental Sciences, vol. 5, no. 1, pp. 197-216, 2014.

[48] H. Zhou, W. T. Yang, X. Zhou et al., "Accumulation of heavy metals in vegetable species planted in contaminated soils and the health risk assessment," International Journal of Environmental Research and Public Health, vol. 13, no. 3, p. 289, 2016.

[49] X. Han, X. Lu, Qinggeletu, and Y. Wu, "Health risks and contamination levels of heavy metals in dusts from parks and squares of an industrial city in semi-arid area of China," International Journal of Environmental Research and Public Health, vol. 14, no. 8, p. 886, 2017.

[50] L. Pan, Y. Wang, J. Ma et al., "A review of heavy metal pollution levels and health risk assessment of urban soils in Chinese cities," Environmental Science and Pollution Research, vol. 25, no. 2, pp. 1055-1069, 2018.

[51] E. Sabath and M. L. Robles-Osorio, "Medio ambiente y riñón: Nefrotoxicidad por metales pesados," Nephrology Magazine. Official Organ of the Sociedad Espanola de Nephrologia, vol. 32, no. 3, pp. 279-286, 2012.

[52] P. Dusek, T. Litwin, and A. Członkowska, "Neurologic impairment in Wilson disease," Annals of Translational Medicine, vol. 7, no. S2, pp. S64-S64, 2019.

[53] K. Anitha, S. Namsani, and J. K. Singh, "Removal of heavy metal ions using a functionalized single-walled carbon nano- tube: a molecular dynamics study," The Journal of Physical Chemistry. A, vol. 119, no. 30, pp. 8349-8358, 2015.

[54] C. Y. Ng, A. W. Mohammad, L. Y. Ng, and J. M. Jahim, "Sequential fractionation of value-added coconut products using membrane processes," Journal of Industrial and Engineering Chemistry, vol. 25, pp. 162-167, 2015.

[55] Y. Haggag, H. Samaha, M. Nossair, and A. Mansour, "Some chemical pollutants of water used in broiler chicken farms and their effect on immune response and body weight of chicken," Alexandria Journal of Veterinary Sciences, vol. 48, no. 2, p. 103, 2016.

[56] K. Singh and S. A. Waziri, "Activated carbons precursor to corncob and coconut shell in the remediation of heavy metals from oil refinery wastewater," Journal of Materials and Environmental Science, vol. 2508, no. 7, pp. 657-667, 2019.

[57] G. Liu, M. R. Garrett, P. Men, X. Zhu, G. Perry, and M. A. Smith, "Nanoparticle and other metal chelation therapeutics in Alzheimer disease," Biochimica et Biophysica Acta (BBA) Molecular Basis of Disease, vol. 1741, no. 3, pp. 246-252, 2005.

[58] A. Singh, R. Kukreti, L. Saso, and S. Kukreti, "Oxidative stress: a key modulator in neurodegenerative diseases," Molecules, vol. 24, no. 8, article 1583, 2019.

[59] World Health Organization Food, Evaluation of Certain Food Additives and Contaminants, vol. 48Geneva, 2nd edition, 2002.

[60] A. Matthews, A. Grimaldi, M. Walker, E. Bartowsky, P. Grbin, and V. Jiranek, "Mathematical modeling for the simulation of heavy metal," Applied and Environmental Microbiology, vol. 5, no. 1, pp. 153-159, 2010.

[61] T. Liu, Y. L. Li, J. Y. He et al., "Porous boron nitride nanoribbons with large width as superior adsorbents for rapid removal of cadmium and copper ions from water," New Journal of Chemistry, vol. 43, no. 8, pp. 3280-3290, 2019.

[62] M. Tuzen, K. O. Saygi, and M. Soylak, "Solid phase extraction of heavy metal ions in environmental samples on multiwalled carbon nanotubes," Journal of Hazardous Materials, vol. 152, no. 2, pp. 632-639, 2008.

[63] H. Huang, T. Chen, X. Liu, and H. Ma, "Ultrasensitive and simultaneous detection of heavy metal ions based on threedimensional graphene-carbon nanotubes hybrid electrode materials," Analytica Chimica Acta, vol. 852, pp. 45-54, 2014.

[64] A. V. Samrot, C. S. Sahithya, J. Selvarani A, S. Pachiyappan, and S. Kumar S, "Surface-engineered super-paramagnetic iron oxide nanoparticles for chromium removal," International Journal of Nanomedicine, vol. 14, pp. 8105-8119, 2019.

[65] Ihsanullah, A. Abbas, A. M. al-Amer et al., "Heavy metal removal from aqueous solution by advanced carbon nanotubes: critical review of adsorption applications," Separation and Purification Technology, vol. 157, pp. 141-161, 2016.

[66] X. Wei, X. Kong, S. Wang, H. Xiang, J. Wang, and J. Chen, "Removal of heavy metals from electroplating wastewater by thin-film composite nanofiltration hollow-fiber membranes," Industrial and Engineering Chemistry Research, vol. 52, no. 49, pp. 17583-17590, 2013.

[67] L. Scalfi, G. Fraux, A. Boutin, and F. X. Coudert, "Structure and dynamics of water confined in imogolite nanotubes," The Journal of Physical Chemistry, vol. 34, no. 23, pp. 21032108, 2018.

[68] B. K. M. A. K. Yadav, R. Abbassi, N. Kumar, S. Satya, and T. R. Sreekrishnan, "The removal of heavy metals in wetland microcosms: effects of bed depth, plant species, and metal 
mobility," International Journal of Chemical Engineering, vol. 1, pp. 501-507, 2012.

[69] H. Badjian and A. R. Setoodeh, "Improved tensile and buckling behavior of defected carbon nanotubes utilizing boron nitride coating - a molecular dynamic study," Physica B: Condensed Matter, vol. 507, pp. 156-163, 2017.

[70] J. Wang, J. Hao, D. Liu et al., "Porous boron carbon nitride nanosheets as efficient metal-free catalysts for the oxygen reduction reaction in both alkaline and acidic solutions," ACS Energy Letters, vol. 2, no. 2, pp. 306-312, 2017.

[71] A. Panahi, A. Shomali, M. H. Sabour, and E. Ghafar-Zadeh, "Molecular dynamics simulation of electric field driven water and heavy metals transport through fluorinated carbon nanotubes," Journal of Molecular Liquids, vol. 278, pp. 658-671, 2019.

[72] S. F. Lim and A. Y. W. Lee, "Kinetic study on removal of heavy metal ions from aqueous solution by using soil," Environmental Science and Pollution Research, vol. 22, no. 13, pp. 10144-10158, 2015.

[73] M. P. Lopes, C. T. Matos, V. J. Pereira et al., "Production of drinking water using a multi-barrier approach integrating nanofiltration: a pilot scale study," Separation and Purification Technology, vol. 119, pp. 112-122, 2013.

[74] A. Khosravanipour Mostafazadeh, M. Zolfaghari, and P. Drogui, "Electrofiltration technique for water and wastewater treatment and bio- products management: a review," Journal of Water Process Engineering, vol. 14, pp. 28-40, 2016.

[75] X. Wei, X. Kong, C. Sun, and J. Chen, "Characterization and application of a thin-film composite nanofiltration hollow fiber membrane for dye desalination and concentration," Chemical Engineering Journal, vol. 223, pp. 172-182, 2013.

[76] J. J. Shang, Q. S. Yang, X. H. Yan, X. Q. He, and K. M. Liew, "Ionic adsorption and desorption of CNT nanoropes," Nanomaterials, vol. 6, no. 10, p. 177, 2016.

[77] Y. Yang, S. Qiao, R. Jin, J. Zhou, and X. Quan, “Anti-fouling characteristic of carbon nanotubes hollow fiber membranes by filtering natural organic pollutants," Korean Journal of Chemical Engineering, vol. 35, no. 4, pp. 964-973, 2018.

[78] O. S. Lee, "Dynamic properties of water confined in graphene-based membrane: a classical molecular dynamics simulation study," Membranes, vol. 9, no. 12, p. 165, 2019.

[79] L. G. Silva, A. M. J. C. Neto, L. Gaffo, R. S. Borges, T. C. Ramalho, and N. Machado, "Molecular dynamics of film formation of metal tetrasulfonated phthalocyanine and poly amidoamine dendrimers," Journal of Nanomaterials, vol. 2013, Article ID 816285, 7 pages, 2013.

[80] M. C. Gordillo, G. Nagy, and J. Martí, "Structure of water nanoconfined between hydrophobic surfaces," The Journal of Chemical Physics, vol. 123, no. 5, article 054707, 2005.

[81] G. Cassone, P. V. Giaquinta, F. Saija, and A. M. Saitta, "Proton conduction in water ices under an electric field," The Journal of Physical Chemistry. B, vol. 118, no. 16, pp. 44194424, 2014.

[82] Z. Fu, Y. Luo, J. Ma, and G. Wei, "Phase transition of nanotube-confined water driven by electric field," The Journal of Chemical Physics, vol. 134, no. 15, article 154507, 2011.

[83] S. P. Shi, Q. Zhang, L. Zhang et al., "Geometrical structures, vibrational frequencies, force constants and dissociation energies of isotopic water molecules $\left(\mathrm{H}_{2} \mathrm{O}, \mathrm{HDO}, \mathrm{D}_{2} \mathrm{O}\right.$, HTO, DTO, and $\mathrm{T}_{2} \mathrm{O}$ ) under dipole electric field," Chinese Physics B, vol. 20, no. 6, article 063102, 2011.
[84] M. J. McAllister, J. L. Li, D. H. Adamson et al., "Single sheet functionalized graphene by oxidation and thermal expansion of graphite," Chemistry of Materials, vol. 19, no. 18, pp. 43964404, 2007.

[85] D. Kaliannan, S. Palaninaicker, V. Palanivel, M. A. Mahadeo, B. N. Ravindra, and S. Jae-Jin, "A novel approach to preparation of nano-adsorbent from agricultural wastes (Saccharum officinarum leaves) and its environmental application," Environmental Science and Pollution Research, vol. 26, no. 6, pp. 5305-5314, 2019.

[86] G. Li, Z. Zhao, J. Liu, and G. Jiang, "Effective heavy metal removal from aqueous systems by thiol functionalized magnetic mesoporous silica," Journal of Hazardous Materials, vol. 192, no. 1, pp. 277-283, 2011.

[87] A. M. Saitta, F. Saija, and P. V. Giaquinta, "Ab initio molecular dynamics study of dissociation of water under an electric field," Physical Review Letters, vol. 108, no. 20, article 207801, 2012.

[88] X. Yang, M. Feng, Y. Chen, H. Lu, and X. Zhou, "Fluid flow in charged nanotubes," Theoretical and Applied Mechanics Letters, vol. 3, no. 3, article 032008, 2013.

[89] M. J. Uline and D. S. Corti, "Molecular dynamics at constant pressure: allowing the system to control volume fluctuations via a 'shell' particle," Entropy, vol. 15, no. 12, pp. 39413969, 2013.

[90] R. J. Mashl, S. Joseph, N. R. Aluru, and E. Jakobsson, “Anomalously immobilized water: a new water phase induced by confinement in nanotubes," Nano Letters, vol. 3, no. 5, pp. 589-592, 2003.

[91] Winarto, E. Yamamoto, and K. Yasuoka, "Water molecules in a carbon nanotube under an applied electric field at various temperatures and pressures," Water, vol. 9, no. 7, p. 473, 2017.

[92] C. Y. Won and N. R. Aluru, "Structure and dynamics of water confined in a boron nitride nanotube," Journal of Physical Chemistry C, vol. 112, no. 6, pp. 1812-1818, 2008.

[93] S. Yu, X. Wang, H. Pang et al., "Boron nitride-based materials for the removal of pollutants from aqueous solutions: a review," Chemical Engineering Journal, vol. 333, pp. 343360, 2018.

[94] T. A. Pascal, W. A. Goddard, and Y. Jung, "Entropy and the driving force for the filling of carbon nanotubes with water," Proceedings of the National Academy of Sciences of the United States of America, vol. 108, no. 29, pp. 11794-11798, 2011.

[95] S. B. Zhu and G. W. Robinson, "Structure and dynamics of liquid water between plates," The Journal of Chemical Physics, vol. 94, no. 2, pp. 1403-1410, 1991.

[96] F. Robinson, M. Shahbabaei, and D. Kim, "Deformation effect on water transport through nanotubes," Energies, vol. 12, no. 23, article 4424, 2019.

[97] A. A. Dezfoli, M. A. Mehrabian, and H. Hashemipour, "Molecular dynamics simulation of heavy metal ions in aqueous solution using Lennard-Jones 12-6 potential," Chemical Engineering Journal, vol. 202, no. 12, pp. 1685-1692, 2015.

[98] F. Hofbauer and I. Frank, "Electrolysis of water in the diffusion layer: first-principles molecular dynamics simulation," Chemistry - A European Journal, vol. 18, no. 1, pp. 277-282, 2012.

[99] C. R. P. Patel, P. Tripathi, A. K. Vishwakarma et al., "Enhanced hydrogen generation by water electrolysis employing carbon nano-structure composites," International 
Journal of Hydrogen Energy, vol. 43, no. 6, pp. 3180-3189, 2018.

[100] P. K. Dubey, A. S. K. Sinha, S. Talapatra, N. Koratkar, P. M. Ajayan, and O. N. Srivastava, "Hydrogen generation by water electrolysis using carbon nanotube anode," International Journal of Hydrogen Energy, vol. 35, no. 9, pp. 3945-3950, 2010.

[101] T. Steiner, "The hydrogen bond in the solid state," Angewandte Chemie International Edition, vol. 41, no. 1, pp. 4876, 2002.

[102] D. Van Der Spoel, P. J. Van Maaren, and H. J. C. Berendsen, "A systematic study of water models for molecular simulation: derivation of water models optimized for use with a reaction field," The Journal of Chemical Physics, vol. 108, no. 24, pp. 10220-10230, 1998.

[103] Z. Wang, J. Zhao, and Q. Cai, " $\mathrm{CO}_{2}$ electroreduction performance of a single transition metal atom supported on porphyrin-like graphene: a computational study," Physical Chemistry Chemical Physics, vol. 19, no. 34, pp. 2311323121, 2017. 